\title{
Stenotrophomonas maltophilia bacteremia in children: risk factors and mortality rate
}

\author{
Mohammed Alsuhaibani ${ }^{* *}$, Alanoud Aljarbou², Sahar Althawadi ${ }^{3}$, Abdulrahman Alsweed ${ }^{4}$ \\ and Sami Al-Hajjar ${ }^{4}$
}

\begin{abstract}
Purpose: Stenotrophomonas maltophilia (S. maltophilia) is an opportunistic and nosocomial pathogen that can cause an invasive and fatal infection, particularly in hospitalized and immunocompromised patients. However, little is known about the impact of $\mathrm{S}$. maltophilia bacteremia in pediatric patients. Therefore, we aimed to identify risk factors for mortality, antibiotics susceptibility to S. maltophilia, and mortality rates in pediatric patients with S. maltophilia bacteremia.

Methods: We conducted a retrospective cohort study by identifying all S. maltophilia positive blood cultures in the microbiology laboratory database between January 2007 and December 2018 from hospitalized pediatric patients (age 1-14 years). After identifying patients with S. maltophilia bacteremia, medical charts were reviewed for demographics, clinical data, and outcomes within seven days of bacteremia diagnosis. Risk factors associated with mortality in S. maltophilia bacteremia patients were determined using univariate and multivariate analyses.
\end{abstract}

Findings: Sixty-eight pediatric patients with S. maltophilia bacteremia were identified. All infections were nosocomial infections, and (88.2\%) bacteremia cases were catheter-related bloodstream infections. On multivariate analysis, ICU admission prior to bacteremia episode and neutropenia were the major risk factors associated with mortality. S. maltophilia was the most susceptible to trimethoprim and sulfamethoxazole (TMP/SMX, 94.1\%), followed by levofloxacin (85.7\%). The overall mortality rate within seven days of S. maltophilia bacteremia diagnosis was 33.8\%.

Conclusion: S. maltophilia bacteremia is a devastating emerging infection associated with high mortality among hospitalized children. Therefore, early diagnosis and prompt management based on local susceptibility data are crucial. Various risk factors, especially ICU admission prior to bacteremia episode and neutropenia, are associated with $S$. maltophilia bacteremia mortality.

Keywords: Trimethoprim-sulfamethoxazole, Stenotrophomonas maltophilia, Bacteremia, Bloodstream infections, Children

\section{Introduction}

Stenotrophomonas maltophilia (S. maltophilia) is a non-fermenting, gram-negative bacillus that can cause opportunistic infections, especially in hospitalized and immunocompromised patients $[1,2]$ This organism could lead to invasive diseases, such as pulmonary, urinary,

*Correspondence: msuhaibani@qumed.edu.sa

${ }^{1}$ Department of Pediatrics, College of Medicine, Qassim University, Qassim 51452, Saudi Arabia

Full list of author information is available at the end of the article gastrointestinal, and soft tissue infections. Additionally, meningitis and endocarditis have been reportedly caused by S. maltophilia infections [3-6]. S. maltophilia has also been recognized as a cause of bacteremia, particularly in intensive care units (ICUs) and among immunocompromised patients. Central venous catheters, using antibiotics, particularly meropenem, lengthy hospitalization, and malignancy have been described as risk factors for $S$. maltophilia bacteremia $[7,8]$.

The intrinsic resistance of S. maltophilia to multiple antibiotics, including cephalosporins and meropenem, original author(s) and the source, provide a link to the Creative Commons licence, and indicate if changes were made. The images or other third party material in this article are included in the article's Creative Commons licence, unless indicated otherwise in a credit line to the material. If material is not included in the article's Creative Commons licence and your intended use is not permitted by statutory regulation or exceeds the permitted use, you will need to obtain permission directly from the copyright holder. To view a copy of this licence, visit http://creativecommons.org/licenses/by/4.0/. The Creative Commons Public Domain Dedication waiver (http://creativeco mmons.org/publicdomain/zero/1.0/) applies to the data made available in this article, unless otherwise stated in a credit line to the data. 
which are commonly used for empiric therapy, makes it a therapeutic challenge. Trimethoprim/sulfamethoxazole (TMP/SMX) is the most effective antibiotic for $S$. maltophilia treatment, but resistance has been reported [9, 10].

S. maltophilia strains are resistant to several antibiotics, which make it a treatment challenge and life-threatening infection. Mortality can be as high as $69 \%$ but varies according to the associated risk factors $[11,12]$ in Saudi Arabia, Studies describing S. maltophilia bacteremia in children are limited $[13,14]$. This study aimed to identify the risk factors for mortality from S. maltophilia bacteremia, determine the antibiotic susceptibility profile of S. maltophilia, and determine the mortality rate in children with $S$. maltophilia bacteremia.

\section{Methods}

This study was a retrospective chart review of children who had been admitted to King Faisal Specialist Hospital and Research Center (KFSHRC) in Riyadh with S. maltophilia bacteremia. KFSHRC is a tertiary care center with transplantation and oncology services. This study was approved by the KFSHRC Institutional Review Board.

All S. maltophilia positive blood cultures between January 2007 and December 2018 among hospitalized children $\leq 14$ years old were included in this study. We identified S. maltophilia-positive blood cultures from the microbiology laboratory database then clinical records were reviewed. Bacteremia was defined as $\geq$ one positive blood culture from a central line or a peripheral blood sample with clinical symptoms $[15,16]$. Asymptomatic patients with positive blood cultures whose $S$. maltophilia bacteremia resolved without treatment (confirmed by negative culture) were excluded because of the possibility of contamination. Hospital-acquired S. maltophilia infection was determined by an isolate recovered from blood culture $48 \mathrm{~h}$ after admission to the hospital [17-19]. Furthermore, isolation of additional bacteria species from the initial blood culture was considered a polymicrobial infection.

We collected the following clinical data: primary diagnosis; admission to the ICU or intubation prior to the bacteremia episode; prior or concomitant use of antibiotics, steroids, chemotherapy, or immunosuppressive therapy; and neutropenia (total granulocyte count $<1000 /$ $\mathrm{mL}$ ) within the 14 days preceding infection. Additionally, the presence of a central line catheter, clinical manifestations and complete blood counts on the first day of bacteremia were recorded. The mortality was defined as death taking place within 7 days from an S. maltophiliapositive blood culture, regardless of comorbidities [7, 20].

All cultures were analyzed using local hospital and laboratory protocols. From 2007 to 2015, the automated
BacTec 9240 (Becton Dickinson, Sparks, MD, USA) was used for blood counts. From 2016 to 2018, BACT/ ALERT $^{\circledR}$ VIRTUO $^{\circledR}$ blood culture detection system (bioMérieux, Marcy l'Étoile, France) was used. S. maltophilia was identified and susceptibility testing was performed using a VITEK ${ }^{\circledR} 2$ (bioMérieux). Antibiotic susceptibility testing was performed using Gram-Negative Card 292. Interpretation of zone diameters for classification as susceptible, intermediate, or resistant was based on the Clinical and Laboratory Standards Institute guidelines (CLSI M100) [21].

Both descriptive and inferential statistics were used for data analysis. Statistical analysis was carried out using Statistical Package for the Social Sciences (SPSS) version 21.0 (IBM Corp, Armonk, NY, USA). The variables were taken as significant at a $p$ value $\leq 0.05$. The associations between independent variables (baseline characteristics, i.e., sex, age, primary diagnosis, malignancy, ICU admission prior to episode, and types of antibiotics) and dependent variables (the outcome), were tested using Chi-square tests. Multivariate regression analysis was used to determine factors independently associated with the outcome of the mortality, and the results were reported as adjusted odds ratios with 95\% CI.

\section{Results}

Seventy-two pediatric patients with S. maltophilia bacteremia were identified. Of the 72 cases, 4 patients were excluded: 1 patient was older than 14 years, and 3 patients had bacteremia that resolved without treatment. The patient demographic and clinical characteristics, and the outcomes are shown in Table 1 . Of the remaining 68 patients, 34 (50\%) were males. The median age was 21.5 months (IQR 5.5-81). The patients' ages were classified as follows: $\leq 12$ months 26 (38.2\%); 13-36 months 21 (30.9\%); and > 36 months 21 (30.9\%), and all the patients developed the bacteremia as hospital-acquired infections.

The most common underlying primary diagnosis was malignancy $20(29.4 \%)$, congenital heart diseases 10 (16.2\%), anemia $(11,14.7 \%)$, and primary immunodeficiency 8 (11.8\%). There were 22 patients $(32.4 \%)$ who had had a transplant, 20 patients $(29.4 \%)$ were on chemotherapy, and 19 patients (27.9\%) were on steroids. The majority of the patients had a central line catheter (97\%), and most of the bacteremia was catheter-related bloodstream infection (88.2\%).

The most common clinical presentation of S. maltophilia bacteremia, fever, was present in $67.6 \%$ of patients, followed by respiratory symptoms (38.2\%). Positive respiratory culture with $S$. maltophilia was detected in 24 patients (35.3\%), and polymicrobial blood culture was found in 21 patients (30.9\%). Polymicrobial blood culture included 60\% gram-negative (Enterobacter, 
Table 1 Demographic and etiologic characteristics of patients with S. maltophilia bacteremia

\begin{tabular}{llll}
\hline Parameters & Overall & Death & Resolved $p$ value $^{\S}$ \\
& $n(\%)$ & $n(\%)$ & $n(\%)$ \\
& $(n=68)$ & $(n=23)$ & $(n=45)$
\end{tabular}

\begin{tabular}{lllll}
\hline Sex & & & & \\
Male & $34(50.0 \%)$ & $13(56.5 \%)$ & $21(46.7 \%)$ & 0.442 \\
Female & $34(50.0 \%)$ & $10(43.5 \%)$ & $24(53.3 \%)$ & \\
Age in months & & & & \\
$\leq 12$ & $26(38.2 \%)$ & $7(30.4 \%)$ & $19(42.2 \%)$ & 0.639 \\
$13-36$ & $21(30.9 \%)$ & $8(34.8 \%)$ & $13(28.9 \%)$ & \\
$>36$ & $21(30.9 \%)$ & $8(34.8 \%)$ & $13(28.9 \%)$ & \\
Primary diagnosis & & & & \\
Malignancy & $20(29.4 \%)$ & $7(30.4 \%)$ & $13(28.9 \%)$ & 0.895 \\
Anemia & $10(14.7 \%)$ & $2(8.7 \%)$ & $8(17.8 \%)$ & 0.317 \\
Cardiac & $11(16.2 \%)$ & $5(21.7 \%)$ & $6(13.3 \%)$ & 0.373 \\
Primary immunodefi- & $8(11.8 \%)$ & $2(8.7 \%)$ & $6(13.3 \%)$ & 0.574 \\
$\quad$ ciency & & & & \\
Metabolic & $5(7.4 \%)$ & $2(8.7 \%)$ & $3(6.7 \%)$ & 0.762 \\
HLH & $6(8.8 \%)$ & $3(13.0 \%)$ & $3(6.7 \%)$ & 0.380 \\
Renal & $3(4.4 \%)$ & $1(4.3 \%)$ & $2(4.4 \%)$ & 0.985 \\
Other & $5(7.4 \%)$ & $1(4.3 \%)$ & $4(8.9 \%)$ & 0.497 \\
\hline
\end{tabular}

$\S p$ value has been calculated using Chi-square test

** Significant at $p \leq 0.05$

Acinetobacter, Pseudomonas, and Klebsiella species), 20\% gram-positive (Enterococcus, Coagulase-negative Staphylococcus, and Streptococcus species), and 20\% candida species. The most common antibiotics taken within 14 days prior to the S. maltophilia bacteremia diagnosis were vancomycin then meropenem Fig. 1.

Antibiotic susceptibility to TMP/SMX was the highest $(94.1 \%)$, followed by levofloxacin (85.7\%), while ceftazidime and ciprofloxacin susceptibilities were $61.9 \%$ and 50\% respectively, as shown in Fig. 2. The antibiotic susceptibility of the S. maltophilia blood isolates is shown in Fig. 2. The risk factors associated with mortality as determined by univariate analysis are shown in Table 2 . Using multivariate regression analysis, we found that ICU admission prior to episode and neutropenia were the risk factors independently associated with mortality, as shown in Table 3. There were no statistically significant differences in mortality in patients received TMP/ SMX as monotherapy (36 patients) and (11 patients) who received it in combination with other antibiotics (fluoroquinolone, ceftazidime, or an aminoglycoside). The overall mortality rate within 7 days of $S$. maltophilia bacteremia diagnosis was $33.8 \%$.

\section{Discussion}

S. maltophilia bacteremia is a relatively rare but lifethreatening infection, causing significant mortality. In our study, we described the risk factors for mortality in 68 pediatric patients with S. maltophilia bacteremia.

Though S. maltophilia is found in the community [7], in our study, all infections were a hospital-acquired infection. Multiple risk factors for mortality have been identified in previous studies of S. maltophilia infections in children, including prior use of antibiotics, neutropenia, mechanical ventilation, ICU stays, and malignancy [2224]. Also, ICU admission showed to be a risk factor for S. maltophilia bacteremia acquisition [25]. In our study, we documented that ICU admission prior to bacteremia episode and neutropenia are risk factors for mortality in patients with S. maltophilia bacteremia. Approximately half of patients in this study were neutropenic, which may be explained that our center is specialized in immunocompromised and oncology patients.

In the current study, we found that polymicrobial infection was most often caused by other gram-negative bacteria (30.9\%) Enterobacter, Acinetobacter,

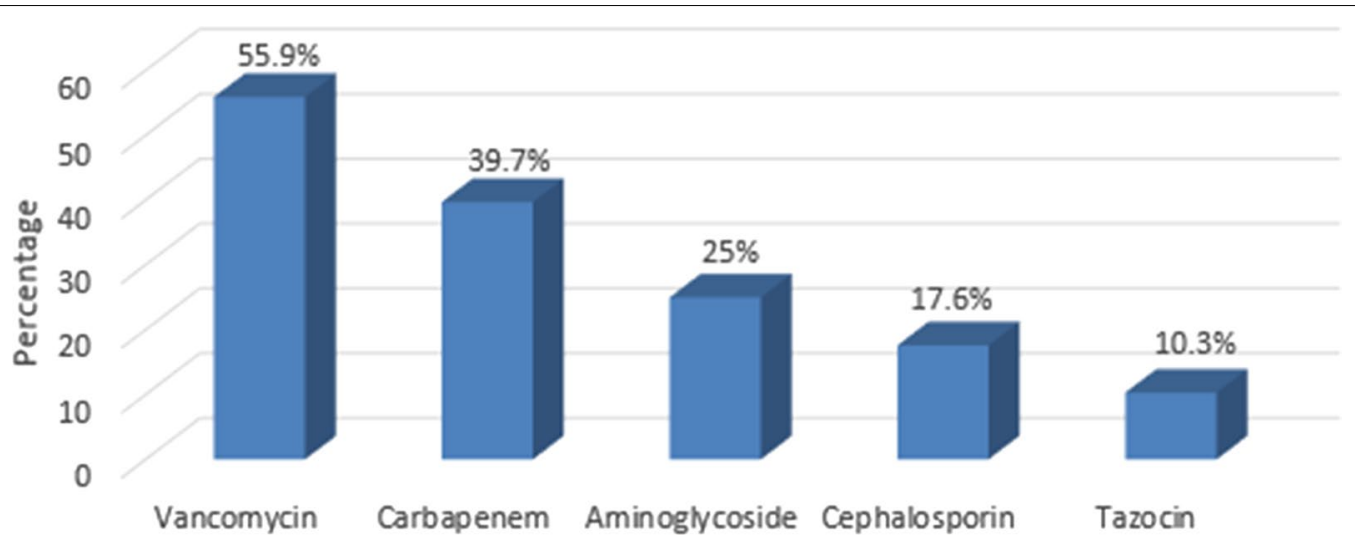

Fig. 1 Antibiotics used prior to S. maltophilia episodes 


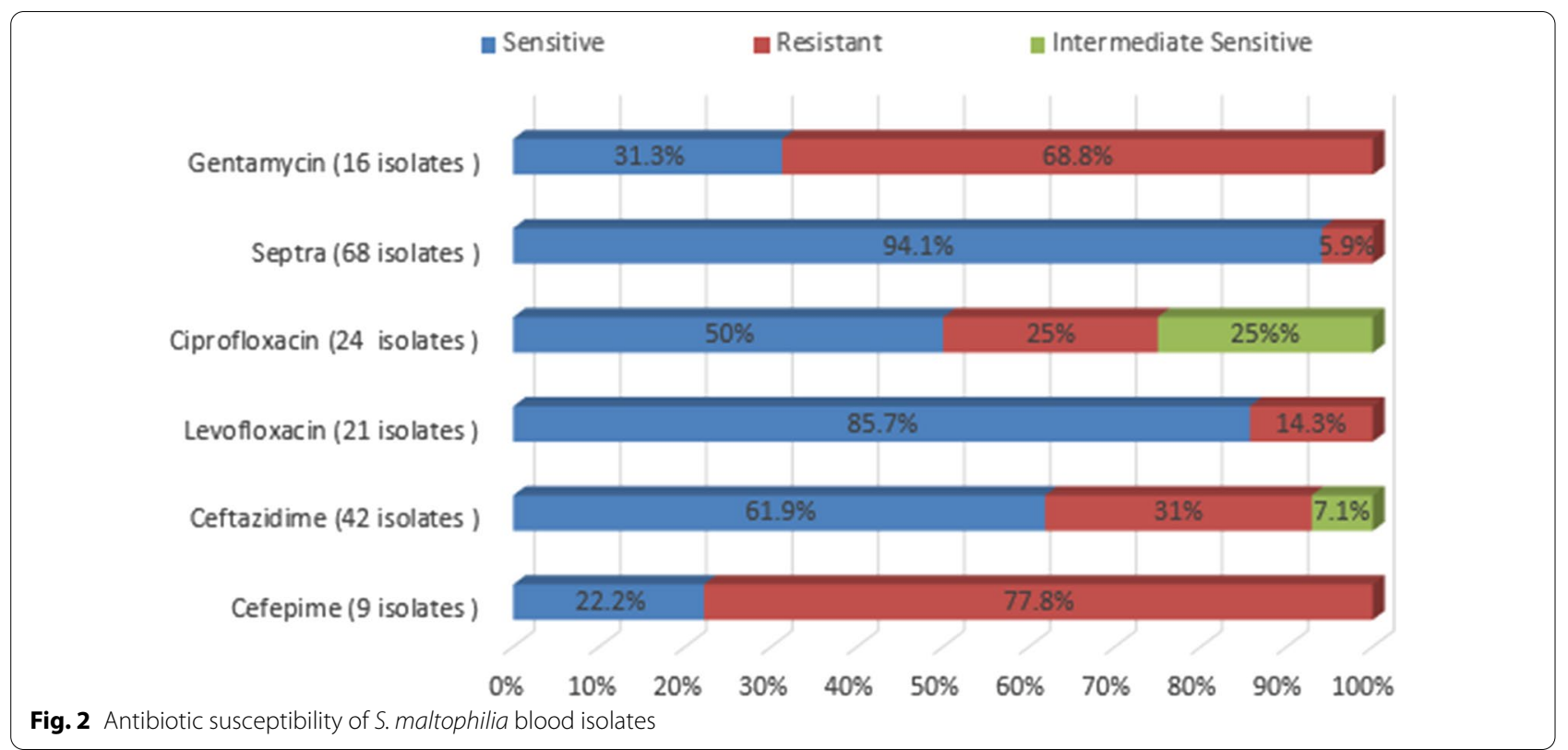

Pseudomonas, and Klebsiella species. Sattler et al. also reported that gram-negative co-infections with Acinetobacter and Enterobacter species were common among children with $S$. maltophilia bacteremia but found a much higher prevalence of polymicrobial infection (70\% vs. 30.9\%) [7].

The management of S. maltophilia bacteremia and sepsis is challenging because of the bacteria's extensive intrinsic and induced antimicrobial resistance [26]. Different molecular mechanisms of resistance have been documented and include $ß$-lactamase production against $ß$-lactam antibiotics, multidrug efflux pumps, the plasmid-encoded qnr gene against quinolones, and the presence of class 1 integrons, known to be responsible for resistance to TMP/SMX [27]. According to SENTRY antimicrobial surveillance data and other literature reviews of bacteremia, most bacterial isolates are highly susceptible to TMP/SMX and levofloxacin, with low to moderate sensitivity to ceftazidime and ciprofloxacin. These data are consistent with the sensitivity pattern among our $S$. maltophilia isolates [28, 29]. Although there is developing resistance to TMP/SMX, the prevalence of resistance in our isolates was very low (5.9\%) which made it the best empirical treatment for our center [30]. Furthermore, we did not find a statistically significant difference in mortality in patients who received TMP/SMX as monotherapy and those who received it in combination with ceftazidime, aminoglycoside or fluoroquinolones. Tokatly et al. advocated the use of a combination of TMP/SMX and ciprofloxacin or minocycline in critically ill children [24]. However, the best empirical or combination treatment for $S$. maltophilia needs further study.

Mortality related to bacteremia in children has been reported to be high, with crude mortality rates ranging from 14 to $69 \%[31,32]$. A 5-year multicenter retrospective study of critically ill children with $S$. maltophilia bacteremia, conducted between 2012 and 2017 , found a crude mortality rate of $42 \%$, although the mortality attributed to infection was $18 \%$ [24]. Our study revealed a 7 -day crude mortality rate of $33.8 \%$, but this decreased to $26.4 \%$ if polymicrobial infections were excluded. A similar study conducted in adults found a crude mortality rate of $47.5 \%$ among patients with S. maltophilia infection and an eightfold increase in mortality risk with an attributable mortality rate of $26.7 \%$ due to bacteremia caused by S. maltophilia [33].

Our study's limitations are its retrospective design, single-center location, the lack of appropriate control groups, and the clinical status of our patients (with many being immunocompromised or in critical care).

This study revealed that $S$. maltophilia bacteremia is an emerging fatal infection associated with high mortality among children. Therefore, early diagnosis and prompt management based on local data susceptibility are crucial. It identified various risk factors associated with S. maltophilia bacteremia mortality, of which ICU admission prior to bacteremia episode and neutropenia were associated with the highest risk. A multicenter, prospective cohort study is needed to confirm these risk factors associated with $S$. maltophilia mortality in children. 
Table 2 Univariate analysis for factors associated with mortality of patients with S. maltophilia bacteremia

\begin{tabular}{|c|c|c|c|c|}
\hline Factor & $\begin{array}{l}\text { Overall } \\
n(\%) \\
(n=68)\end{array}$ & $\begin{array}{l}\text { Death } \\
n(\%) \\
(n=23)\end{array}$ & $\begin{array}{l}\text { Resolved } \\
n(\%) \\
(n=45)\end{array}$ & $p$ value ${ }^{\S}$ \\
\hline \multicolumn{5}{|c|}{ ICU admission prior to episode } \\
\hline Yes & $28(41.2 \%)$ & $19(82.6 \%)$ & $9(20 \%)$ & \multirow[t]{2}{*}{$<0.001^{* *}$} \\
\hline No & $40(58.8 \%)$ & $4(17.4 \%)$ & $36(80 \%)$ & \\
\hline \multicolumn{5}{|c|}{ Intubation prior to episode } \\
\hline Yes & $23(33.8 \%)$ & $14(60.9 \%)$ & $9(20.0 \%)$ & \\
\hline No & $45(66.2 \%)$ & $9(39.1 \%)$ & $36(80.0 \%)$ & \\
\hline \multicolumn{5}{|c|}{ Immunosuppressive therapy } \\
\hline Yes & $22(32.4 \%)$ & $7(30.4 \%)$ & $15(33.3 \%)$ & \multirow[t]{2}{*}{0.809} \\
\hline No & $46(67.6 \%)$ & $16(69.6 \%)$ & $30(66.7 \%)$ & \\
\hline \multicolumn{5}{|l|}{ Chemotherapy } \\
\hline Yes & $20(29.4 \%)$ & $7(30.4 \%)$ & $13(28.9 \%)$ & \multirow[t]{2}{*}{0.895} \\
\hline No & $48(70.6 \%)$ & $16(69.6 \%)$ & $32(71.1 \%)$ & \\
\hline \multicolumn{5}{|c|}{ Received meropenem } \\
\hline Yes & $27(39.7 \%)$ & $15(65.2 \%)$ & $12(26.7 \%)$ & \\
\hline No & $41(60.3 \%)$ & $8(34.8 \%)$ & $33(73.3 \%)$ & \\
\hline \multicolumn{5}{|l|}{ Organ transplant } \\
\hline Yes & $22(32.4 \%)$ & $6(26.1 \%)$ & $16(35.6 \%)$ & \multirow[t]{2}{*}{0.430} \\
\hline No & $46(67.6 \%)$ & $17(73.9 \%)$ & $29(64.4 \%)$ & \\
\hline \multicolumn{5}{|l|}{ Steroid therapy } \\
\hline Yes & $19(27.9 \%)$ & $7(30.4 \%)$ & $12(26.7 \%)$ & \multirow[t]{2}{*}{0.743} \\
\hline No & $49(72.1 \%)$ & $16(69.6 \%)$ & $33(73.3 \%)$ & \\
\hline \multicolumn{5}{|l|}{ Neutropenia } \\
\hline Yes & $32(47.1 \%)$ & $16(69.6 \%)$ & $16(35.6 \%)$ & \\
\hline No & $36(52.9 \%)$ & $7(30.4 \%)$ & $29(64.4 \%)$ & \\
\hline \multicolumn{5}{|l|}{ Central line } \\
\hline Yes & $66(97.1 \%)$ & $23(100.0 \%)$ & $43(95.6 \%)$ & \multirow[t]{2}{*}{0.305} \\
\hline No & $2(2.9 \%)$ & 0 & $2(4.4 \%)$ & \\
\hline \multicolumn{5}{|l|}{ Platelet count } \\
\hline$<150$ & $56(82.4 \%)$ & $23(100 \%)$ & $33(73.3 \%)$ & \\
\hline$\geq 150$ & $12(17.6 \%)$ & 0 & $12(26.7 \%)$ & \\
\hline \multicolumn{5}{|c|}{ Positive respiratory culture } \\
\hline Yes & $24(35.3 \%)$ & $17(73.9 \%)$ & $7(15.6 \%)$ & \multirow[t]{2}{*}{$<0.001^{* *}$} \\
\hline No & $44(64.7 \%)$ & $6(26.1 \%)$ & $38(84.4 \%)$ & \\
\hline \multicolumn{5}{|c|}{ Treatment with TMP/SMX } \\
\hline Monotherapy & $36(76.6 \%)$ & $11(73.3 \%)$ & $25(78.1 \%)$ & \multirow[t]{2}{*}{0.718} \\
\hline Combination & $11(23.4 \%)$ & $4(26.7 \%)$ & 7 (21.9\%) & \\
\hline
\end{tabular}

$\$ p$ value has been calculated using Chi-square test

**Significant at $p \leq 0.05$ 


\section{Table 3 Multivariate regression analysis to predict the influence of non-survival rates from the baseline characteristics of participants $(n=68)$}

\begin{tabular}{llll}
\hline Factor & AOR & $\mathbf{9 5 \% ~ C l}$ & $\boldsymbol{p}$ value $^{\mathbf{\S}}$ \\
\hline ICU admission & & & \\
Yes & Ref & & \\
No & 0.036 & $0.003-0.406$ & \\
Intubation before episodes & & & 0.802 \\
Yes & Ref & & \\
No & 0.742 & $0.072-7.632$ & 0.844 \\
Prior use of antibiotics & & & \\
Yes & Ref & & \\
No & 1.275 & $0.114-14.294$ & \\
Received meropenem & & & \\
Yes & Ref & & \\
No & 0.242 & $0.049-1.185$ & \\
Had neutropenia & & & \\
Yes & Ref & & \\
No & 0.083 & $0.013-0.537$ &
\end{tabular}

$A O R$ adjusted odds ratio, $\mathrm{Cl}$ confidence interval

**Significant at $p \leq 0.05$ level

\section{Authors' contributions}

MA designed the study. AA, AA made data analysis. Literature search and the manuscript drafting done by MA, AA. SA, SA participated in manuscript writing and revision. All authors read and approved the final manuscript.

\section{Funding}

Researchers would like to thank the Deanship of Scientific Research, Qassim University for funding publication of this project.

\section{Availability of data and materials}

The data and material of the study are available from the corresponding author MA on request.

\section{Ethics approval and consent to participate}

The clinical data and laboratory information were retrospectively obtained from patient medical records. Ethics approval was obtained from KFSHRC Institutional Review Board in Riyadh, Saudi Arabia. Informed consent was not required because of the retrospective cohort design of this study, lack of patient intervention and all patients' data were de-identified.

\section{Consent for publication}

Not applicable.

\section{Competing interests}

The authors declare that they have no conflicts of interest.

\section{Author details}

1 Department of Pediatrics, College of Medicine, Qassim University, Qassim 51452, Saudi Arabia. ${ }^{2}$ Department of Pediatrics, College of Medicine, Imam Mohammad Ibn Saud Islamic University, Riyadh, Saudi Arabia. ${ }^{3}$ Department of Pathology and Laboratory Medicine, Microbiology Laboratory, King Faisal Specialist Hospital and Research Center, Riyadh, Saudi Arabia. ${ }^{4}$ Department of Pediatrics, King Faisal Specialist Hospital and Research Center, Riyadh, Saudi Arabia.

Received: 24 September 2020 Accepted: 5 January 2021

Published online: 22 January 2021

\section{References}

1. Denton M, Kerr KG. Microbiological and clinical aspects of infection associated with Stenotrophomonas maltophilia. Clin Microbiol Rev Am Soc Microbiol. 1998; 11:57-80.

2. Brooke JS. Stenotrophomonas maltophilia: an emerging global opportunistic pathogen. Clin Microbiol Rev Am Soc Microbiol. 2012;25:2-41.

3. Looney WJ, Narita M, Mühlemann K. Stenotrophomonas maltophilia: an emerging opportunist human pathogen. Lancet Infect Dis. 2009:9:312-23.

4. Correia CR, Ferreira ST, Nunes P. Stenotrophomonas maltophilia: rare cause of meningitis. Pediatr Int . 2014;56:e21-2.

5. Burns RL, Lowe L. Xanthomonas maltophilia infection presenting as erythematous nodules. J Am Acad Dermatol . 1997;37:836-8.

6. Subhani S, Patnaik AN, Barik R, Nemani L. Infective endocarditis caused by Stenotrophomonas maltophilia: a report of two cases and review of literature. Indian Heart J. 2016:68:S267-70.

7. Sattler CA, Mason EO Jr, Kaplan SL. Nonrespiratory Stenotrophomonas maltophilia infection at a children's hospital. Clin Infect Dis. 2000;31:1321-30

8. Micozzi A, Venditti M, Monaco M, Friedrich A, Taglietti F, Santilli S, et al. Bacteremia due to Stenotrophomonas maltophilia in patients with hematologic malignancies. Clin Infect Dis. 2000;31:705-11.

9. Biagi M, Tan X, Wu T, Jurkovic M, Vialichka A, Meyer K, et al. Activity of potential alternative treatment agents for Stenotrophomonas maltophilia isolates nonsusceptible to levofloxacin and/or trimethoprim-sulfamethoxazole. J Clin Microbiol. 2020;58:e01603-19.

10. Gajdács M, Urbán E. Prevalence and antibiotic resistance of Stenotrophomonas maltophilia in respiratory tract samples: a 10-year epidemiological snapshot. Heal Serv Res Manag Epidemiol. 2019;6:2333392819870774.

11. Juhász E, Pongrácz J, Iván M, Kristóf K. Antibiotic susceptibility of sulfamethoxazole-trimethoprim resistant Stenotrophomonas maltophilia strains isolated at a tertiary care centre in Hungary. Acta Microbiol Immunol Hung Akadémiai Kiadó. 2015;62:295-305.

12. Jeon $Y D$, Jeong WY, Kim MH, Jung IY, Ahn MY, Ann HW, et al. Risk factors for mortality in patients with Stenotrophomonas maltophilia bacteremia. Medicine. 2016:95:e4375.

13. Alqahtani JM. Emergence of Stenotrophomonas maltophilia nosocomial isolates in a Saudi children's hospital: risk factors and clinical characteristics. Saudi Med J. 2017;38:521-7.

14. Naeem T, Absar M, Somily AM. Antibiotic resistance among clinical isolates of Stenotrophomonas maltophilia at a teaching hospital in Riyadh, Saudi Arabia. J Ayub Med Coll Abbottabad Pakistan. 2012;24:30-3.

15. Tomlinson D, Mermel LA, Ethier M-C, Matlow A, Gillmeister B, Sung L. Defining bloodstream infections related to central venous catheters in patients with cancer: a systematic review. Clin Infect Dis. 2011;53:697710. https://doi.org/10.1093/cid/cir523.

16. Velázquez-Acosta C, Zarco-Márquez S, Jiménez-Andrade MC, VolkowFernández P, Cornejo-Juárez P. Stenotrophomonas maltophilia bacteremia and pneumonia at a tertiary-care oncology center: a review of 16 years. Support Care Cancer. 2018;26:1953-60.

17. Zhang Y, Du M, Johnston JM, Andres EB, Suo J, Yao H, et al. Estimating length of stay and inpatient charges attributable to hospital-acquired bloodstream infections. Antimicrob Resist Infect Control. 2020·9·137.

18. Friedman ND, Kaye KS, Stout JE, McGarry SA, Trivette SL, Briggs JP, et al. Health care-associated bloodstream infections in adults: a reason to change the accepted definition of community-acquired infections. Ann Intern Med USA. 2002;137:791-7.

19. Haque M, Sartelli M, McKimm J, Abu BM. Health care-associated infections-an overview. Infect Drug Resist. 2018;11:2321-33.

20. Pittet $D$, Wenzel RP. Nosocomial bloodstream infections. Secular trends in rates, mortality, and contribution to total hospital deaths. Arch Intern Med. 1995:155:1177-84.

21. Institute $C$ and LS. Performance standards for antimicrobial susceptibility testing. CLSI Suppl. M100. Clinical and Laboratory Standards Institute Wayne, PA; 2017.

22. Wu PS, Lu CY, Chang LY, Hsueh PR, Lee PI, Chen JM, et al. Stenotrophomonas maltophilia bacteremia in pediatric patients - a 10-year analysis. J Microbiol Immunol Infect. 2006:39:144-9. 
23. Güriz H, Çiftçi E, Ayberkin E, Aysev D, Ince E, Arsan S, et al. Stenotrophomonas maltophilia bacteraemia in Turkish children. Ann Trop Paediatr. 2008;28:129-36.

24. Tokatly Latzer I, Paret G, Rubinstein M, Keller N, Barkai G, Pessach IM. Management of Stenotrophomonas maltophilia infections in critically ill children. Pediatr Infect Dis J. 2018;37:981-6.

25. Sumida K, Chong Y, Miyake N, Akahoshi T, Yasuda M, Shimono N, et al. Risk factors associated with Stenotrophomonas maltophilia bacteremia: a matched case-control study. PLoS ONE. 2015;10:e0133731-e0133731.

26. Cai B, Tillotson G, Benjumea D, Callahan P, Echols R. The Burden of bloodstream infections due to Stenotrophomonas maltophilia in the United States: a large, retrospective database study. Open Forum Infect Dis. 2020. https://doi.org/10.1093/ofid/ofaa141.

27. Chang YT, Lin CY, Chen YH, Hsueh PR. Update on infections caused by Stenotrophomonas maltophilia with particular attention to resistance mechanisms and therapeutic options. Front Microbiol. 2015;6:1-20.

28. Sader HS, Jones RN, Stilwell MG, Dowzicky MJ, Fritsche TR. Tigecycline activity tested against 26,474 bloodstream infection isolates: a collection from 6 continents. Diagn Microbiol Infect Dis . 2005;52:181-6.

29. Wang YL, Scipione MR, Dubrovskaya Y, Papadopoulos J. Monotherapy with fluoroquinolone or trimethoprim-sulfamethoxazole for treatment of Stenotrophomonas maltophilia infections. Antimicrob Agents Chemother. 2014;58:176-82.
30. Gales AC, Seifert H, Gur D, Castanheira M, Jones RN, Sader HS, Antimicrobial susceptibility of Acinetobacter calcoaceticus-Acinetobacter baumannii complex and Stenotrophomonas maltophilia clinical isolates: results from the SENTRY antimicrobial surveillance program (1997-2016). Open forum Infect Dis. 2019;6:S34-46.

31. Arthur C, Tang X, Romero JR, Gossett JG, Harik N, Prodhan P. Stenotrophomonas maltophilia infection among young children in a cardiac intensive care unit: a single institution experience. Pediatr Cardiol . 2015;36:509-15.

32. Wisplinghoff H. Pseudomonas spp., Acinetobacter spp. and miscellaneous gram-negative bacilli. Infect Dis (Auckl). 2017. https://doi. org/10.1016/B978-0-323-04579-7.00170-2.

33. Senol E, DesJardin J, Stark PC, Barefoot L, Snydman DR. Attributable mortality of Stenotrophomonas maltophilia bacteremia. Clin Infect Dis. 2002:34:1653-6.

\section{Publisher's Note}

Springer Nature remains neutral with regard to jurisdictional claims in published maps and institutional affiliations.
Ready to submit your research? Choose BMC and benefit from:

- fast, convenient online submission

- thorough peer review by experienced researchers in your field

- rapid publication on acceptance

- support for research data, including large and complex data types

- gold Open Access which fosters wider collaboration and increased citations

- maximum visibility for your research: over $100 \mathrm{M}$ website views per year

At BMC, research is always in progress.

Learn more biomedcentral.com/submissions 